\title{
Correction: Depression, mitochondrial bioenergetics, and electroconvulsive therapy: a new approach towards personalized medicine in psychiatric treatment - a short review and current perspective
}

Alexander Karabatsiakis (10) and Carlos Schönfeldt-Lecuona

Correction to: Translational Psychiatry https://doi.org/10.1038/s41398-020-00901-7 published online 9 July 2020

In the original Article, Dr. Karabatsiakis and Prof. Dr. Schönfeldt-Lecuona's names were indexed incorrectly as our system registered their academic titles as part of their names. We have corrected their names in the XML (this change will reflect in Pubmed).

Published online: 10 August 2020 